CASE REPORT

\title{
Two Unique Cases of Takotsubo Syndrome: Asymptomatic Takotsubo Complicated by Massive Left Ventricular Thrombus and Nightmare Induced Takotsubo Syndrome
}

\author{
Robert W Murdock, $\mathrm{DO}^{1^{*}}$, Todd Thomas, $\mathrm{DO}^{1}$ and David K Murdock, MD \\ ${ }^{1}$ MercyOne North lowa Medical Center, USA \\ ${ }^{2}$ Aspirus Research Institute, USA
}

*Corresponding author: Robert W Murdock, DO, MercyOne North lowa Medical Center, $10004^{\text {th }}$ Street Southwest, Mason City, IA 50401, USA

\section{Introduction}

Takotsubo syndrome (TTS) typically presents like an acute coronary syndrome and is commonly associated with acute physical and/or emotional stress [1]. TTS is characterized by transient moderate to severe left ventricular (LV) dysfunction in the absence of obstructive coronary artery disease (CAD) [2]. Excessive beta adrenergic stimulation remains the most widely accepted theory to account for development of TTS $[1,2]$. Importantly a variety of commonly used pharmacologic agents which enhance catecholaminergic activity have been recognized as causing or facilitating TTS [3]. Increasing awareness of precipitating factors and associated complications of TTS has led to a broader understanding of the presentation, clinical course, and pathophysiology of this syndrome. Here we present two cases of TTS; One representing an uncommon complication and another with a unique mechanism of emotional stress.

Asymptomatic Takotsubo Complicated by Massive Left Ventricular Thrombus

A 58-year-old female with history of ulcerative colitis

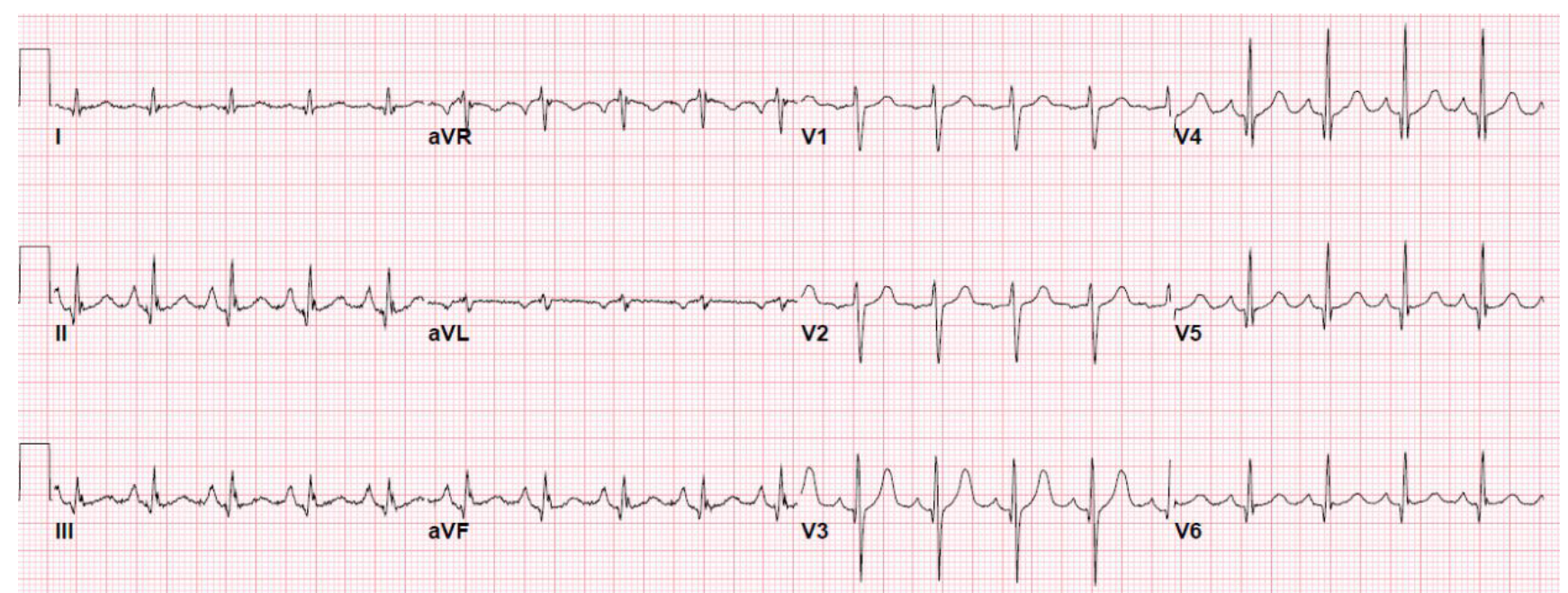

Figure 1: ECG of patient with asymptomatic Takotsubo syndrome (TTS).

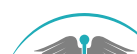

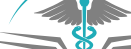

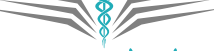

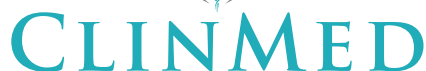

INTERNATIONAL LIBRARY

Citation: Murdock RW, Thomas T, Murdock DK (2019) Two Unique Cases of Takotsubo Syndrome: Asymptomatic Takotsubo Complicated by Massive Left Ventricular Thrombus and Nightmare Induced Takotsubo Syndrome. Int J Clin Cardiol 6:163. doi.org/10.23937/2378-2951/1410163

Accepted: December 11, 2019; Published: December 13, 2019

Copyright: (C) 2019 Murdock RW, et al. This is an open-access article distributed under the terms of the Creative Commons Attribution License, which permits unrestricted use, distribution, and reproduction in any medium, provided the original author and source are credited. 
was being treated for increased symptoms for the preceding month with prednisone and mesalamine. She presented to the emergency department with severe abdominal pain following twelve hours of progressive nausea and generalized abdominal pain. She had no cardiovascular symptoms. Her exam was consistent with acute abdomen. CT scan showed pneumoperitoneum and labs were consistent with peritonitis. Her preoperative ECG showed nonspecific ST segment changes (Figure 1) and she was taken urgently to the operating suite for exploratory laparotomy with repair of a cecal perforation, appendectomy, and loop ileostomy. The initial CT scan of her abdomen and pelvis was also significant for an incidental $5 \mathrm{~cm}$ mass in the LV (Figure 2). Due to this finding cardiology was asked to consult post operatively. A 2D echocar- diogram demonstrated severe apical LV dysfunction with an ejection fraction of $25 \%$. It confirmed a large mass in the LV apex occupying approximately $60 \%$ of the cavity with possible necrotic characteristics concerning for thrombus or neoplasm (Figure 3). She was started on a heparin drip approximately twelve hours later when post-operative hemostasis was secured. A cardiac MRI was consistent with LV thrombus rather than a neoplastic lesion (Figure 4). Her coronary angiography showed mild non-obstructive CAD and a diagnosis of TTS was made. She recovered rapidly from her surgery and after stabilization was started on warfarin, lisinopril, and carvedilol and was discharged when her INR value was therapeutic. Echocardiogram one month later demonstrated significant reduction in thrombus size with moderate improvement of LV

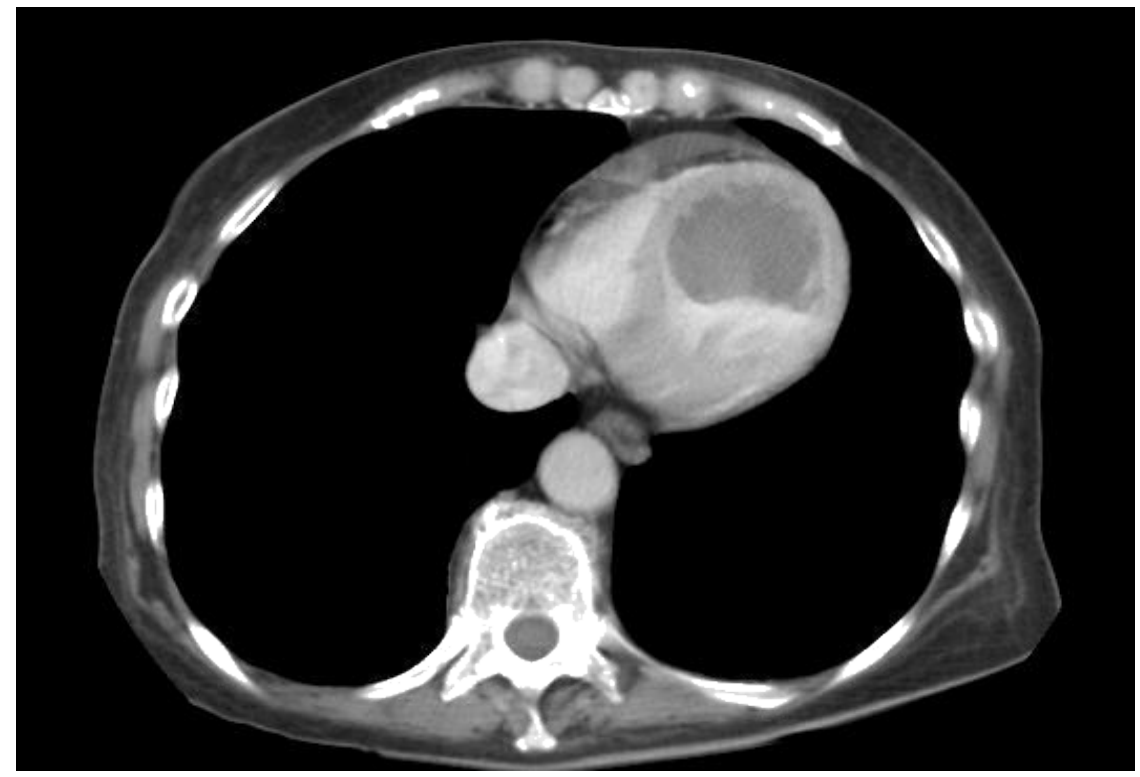

Figure 2: CT scan showing massive left ventricular (LV) thrombus.

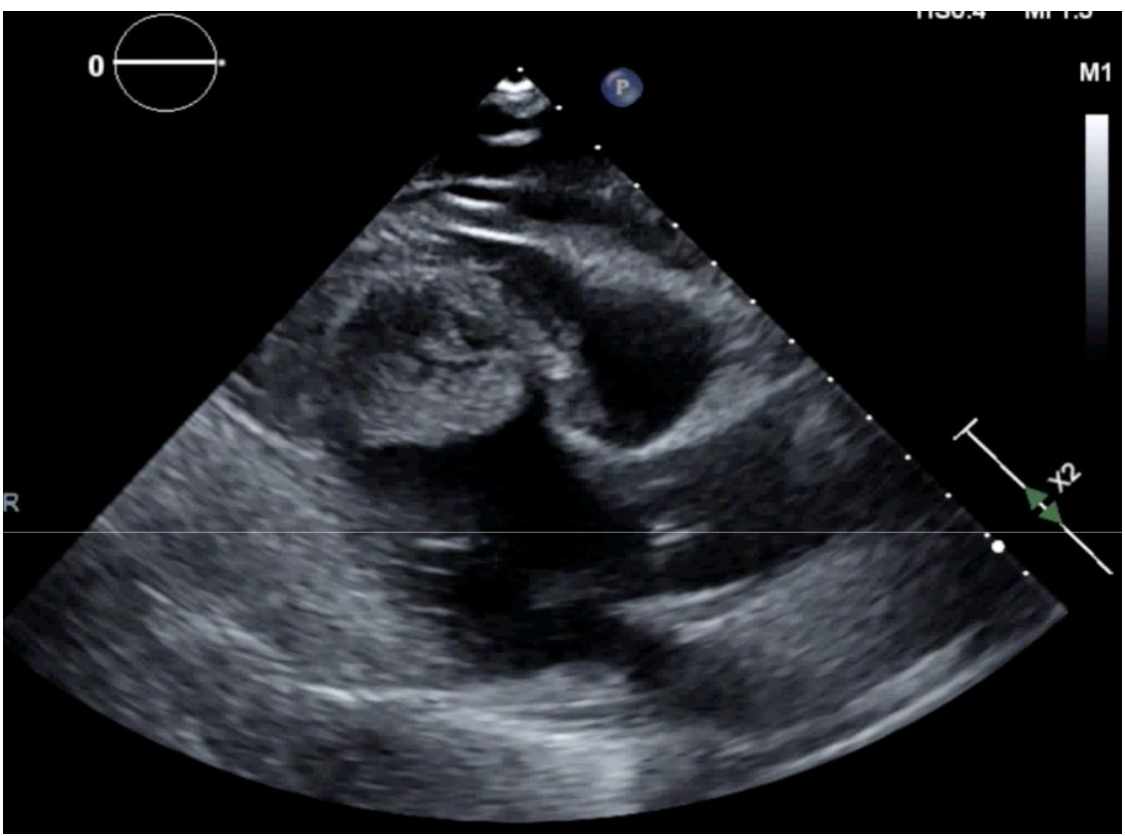

Figure 3: Transthoracic echocardiogram showing apical left ventricular (LV) thrombus in parasternal long axis view. 


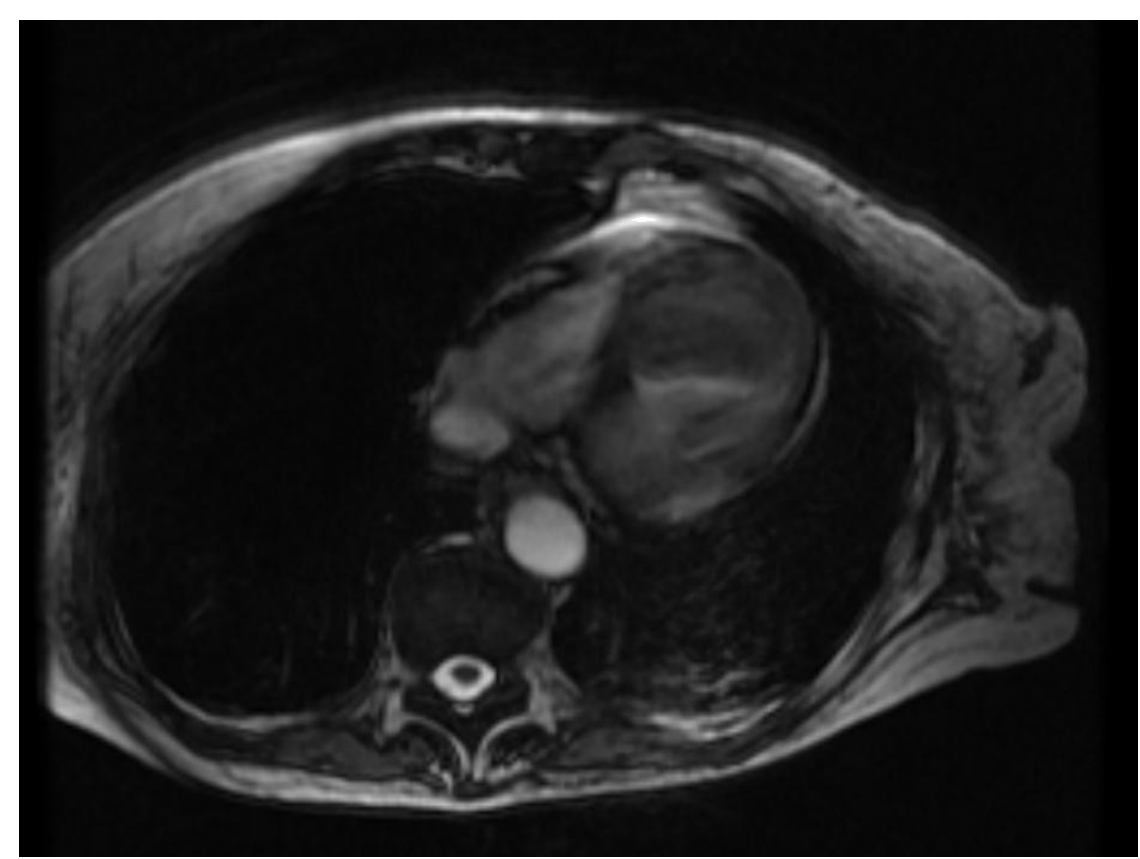

Figure 4: MRI showing non-enhancing avascular apical mass consistent with left ventricular (LV) thrombus.

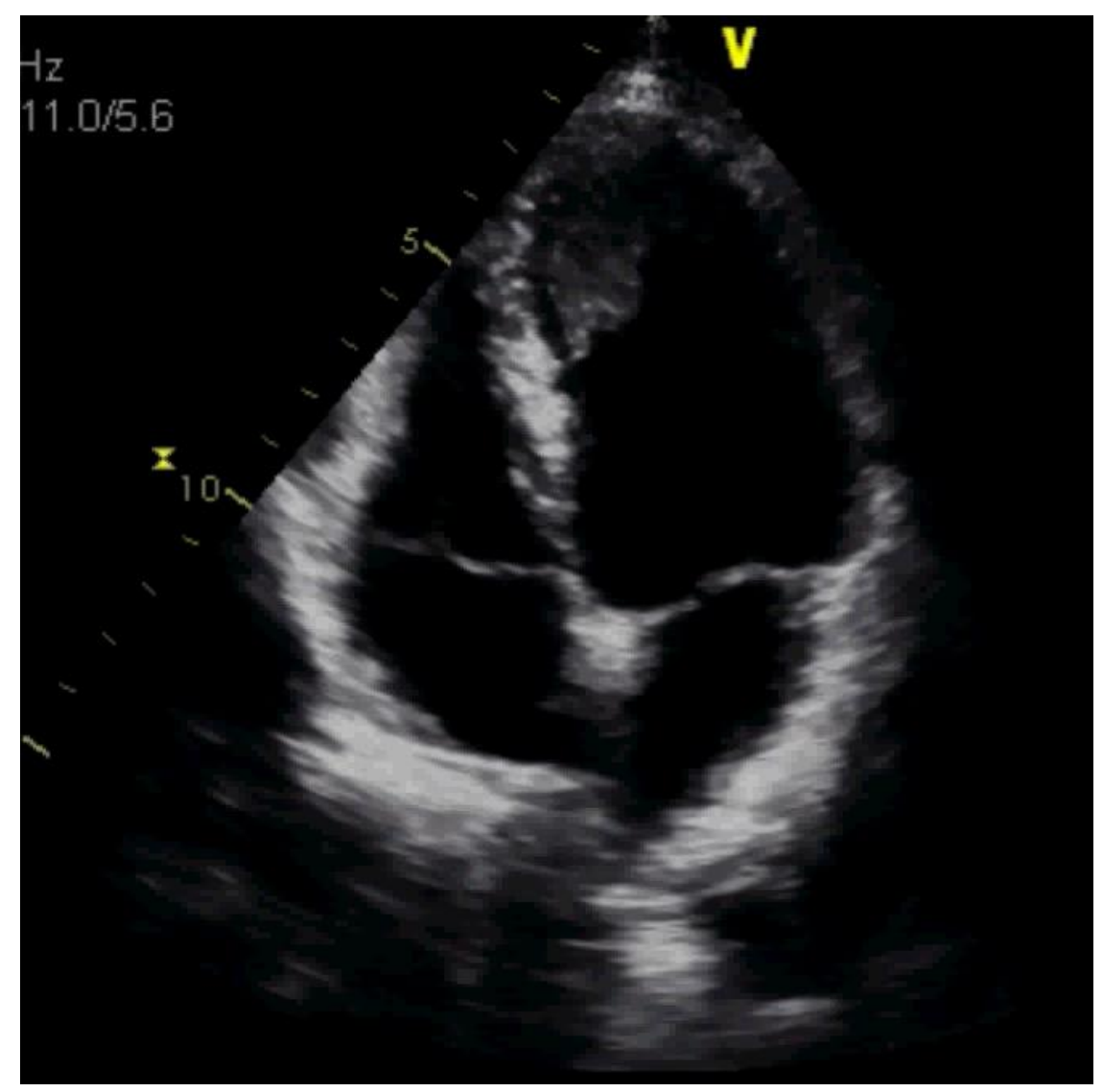

Figure 5: Transthoracic echocardiogram in apical view showing partial thrombus resolution.

function, echocardiogram one additional month later showed complete resolution of the thrombus and normalization of LV function (Figure 5 and Figure 6).

\section{Nightmare Induced TTS}

A 77-year-old woman with a history of generalized depressive anxiety disorder, paroxysmal atrial fibrillation, hypertension, and hyperlipidemia was admit- ted with recurrent TTS in January 2019 after having a prior episode of TTS in June 2017. Her initial episode of TTS occurred at an airport and was likely caused by fear and anxiety that she would miss her connecting flight. At that time coronary angiogram was normal however her left ventriculogram showed marked apical akinesis and an ejection fraction of $25 \%$. She was placed on metoprolol succinate $12.5 \mathrm{mg}$ by daily 

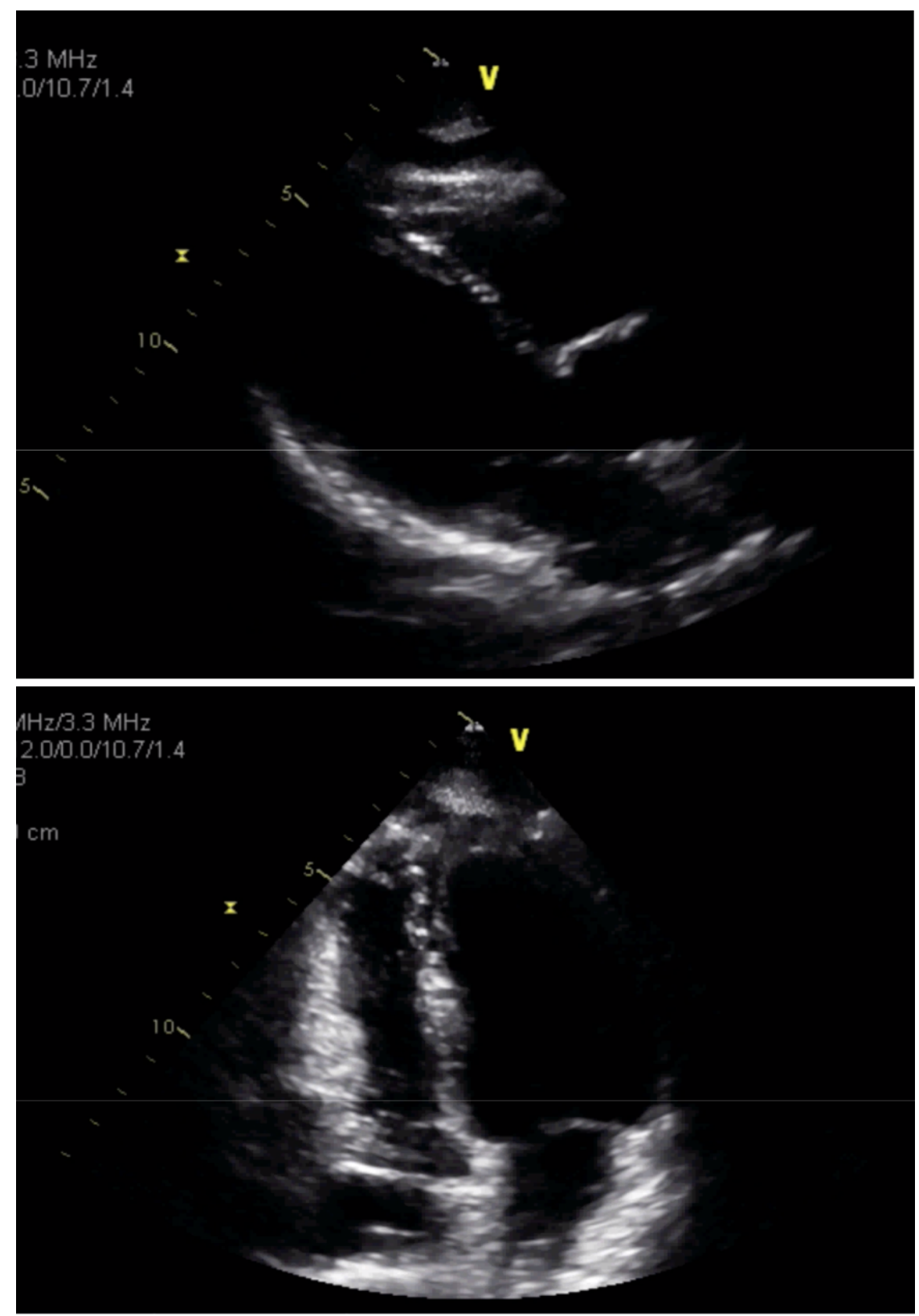

Figure 6: Transthoracic echocardiogram showing resolution of left ventricular (LV) thrombus in parasternal and apical views.

and discharged several days later without incident. Echocardiogram at 3 months showed normal LV wall motion and normal LV ejection fraction, metoprolol succinate was increased to $25 \mathrm{mg}$ daily at that time. She had no further cardiac issues until her presentation to the emergency room in January 2019 with palpitations, diaphoresis, and mild chest discomfort which she experienced upon awaking from a vivid nightmare in which she was being chased and felt her life was threatened. She described her symptoms as similar to those experienced in 2017. Her examination showed blood pressure 155/83, pulse 90 beats per minute, oxygen saturation $96 \%$ on room air, a febrile and without significant physical findings. She was found to have an elevated high sensitivity troponin of $298 \mathrm{ng} / \mathrm{L}$, elevated NT-pro BNP of 2,371 pg/mL, and ECG with normal sinus rhythm and nonspecific $T$ wave changes in the anterior leads. After receiv- ing aspirin $324 \mathrm{mg}$ she was transferred to our center for ongoing care. Her echocardiogram demonstrated severe LV hypokinesis of the apex with ejection fraction of $40-45 \%$. Because of her prior history of normal coronaries, TTS, and described stress prior to this presentation the decision was made to treat her medically and not repeat catheterization. Her metoprolol succinate was changed to carvedilol. Her troponin trended down and she returned to her baseline within 24 hours. She was discharged and follow up echocardiogram 2 weeks later showed normalization of LV systolic function.

\section{Discussion}

Apical LV thrombus is a well known complication of TTS when significant LV dysfunction is present [47]. This is likely cased by stasis from severe wall motion abnormalities as well as a prothrombotic state 
from local tissue injury [8]. Cardioembolic phenomena can occur and is reported in $2-8 \%$ of cases, these primarily happen in patients prior to the institution of systemic anticoagulation. Although large apical LV thrombi have been reported [7,9], to our knowledge this case is by far the most massive thrombus reported with clot burden occupying greater than $60 \%$ of the LV chamber (Figure 2, Figure 3 and Figure 4). We believe that this patient's TTS was caused by severe pain and metabolic stress from her acute abdomen. Our case is additionally unique in that it was discovered incidentally in a patient with no cardiovascular (CV) symptoms or electrocardiographic abnormalities suggestive of an acute coronary syndrome. However we recognize that her severe abdominal symptoms and decompensated state on presentation may have masked more subtle CV symptoms.

We chose to manage our patient with warfarin rather than a novel oral anticoagulant due to lack of evidence supporting use of direct oral anticoagulant agents as well as numerous reports of successful treatment of LV thrombi with warfarin [4-7,9]. Through serial echocardiograms, our case nicely illustrates the progressive resolution of both the LV dysfunction and the apical thrombus (Figure 3, Figure 5 and Figure 6). Although evidence regarding optimal duration of anticoagulation is lacking, current recommendations suggest 3 months or until the wall motion abnormalities are resolved [4]. There is no guideline regarding prophylactic oral anticoagulation in patients with TTS, however it seems reasonable to employ oral anticoagulation in patients with severe wall motion abnormalities until the LV function normalizes.

Depressive anxiety disorder is frequently associated with patients experiencing TTS due to emotional distress $[1,2]$. Our patient with history of generalized anxiety disorder presented with a recurrent episode of TTS following a severe and vividly frightening nightmare. To our knowledge this is a very rare presentation with few reported incidents [10]. Our case adds to the few cases of nightmare induced TTS, all of which occurred in patients with a history of depressive anxiety disorder. As there is a reported predilection for early AM TTS $[1,11,12]$, it is possible that some of these cases may be due to unrecognized nightmare induced TTS.

It is interesting to note that our patient developed recurrent TTS despite being on metoprolol succinate. Indeed as many as $20 \%$ of patients presenting with TTS are on a beta-blocker at the time of initial presentation [13]. There is compelling evidence that TTS results from hyper stimulation of beta 2 receptors causing a stimulatory response to become an inhibitory response $[1,14]$. Theoretically medications which block both beta 1 and beta 2 receptors may be preferred over beta 1 specific agents. For this reason we elect to treat a majority of our TTS patients with carvedilol rather than metoprolol however it should be noted that this approach is based on clinical rationale and remains unproven.

\section{Conclusion}

As knowledge of TTS expands it is increasingly recognized that presentation of the syndrome can vary widely. Our paper adds to the current literature and elucidates unique cases which illustrate potential treatment challenges. Our case of incidentally found TTS with LV thrombus shows that a high index of suspicion must be maintained in patients who may be at risk of TTS. It is highly likely that TTS is under diagnosed in patients experiencing high levels of physiologic or emotional stress. This case also clearly shows that TTS places a patient at risk of thrombotic complications. We believe it's reasonable to consider anticoagulation in any patient with TTS and severe LV dysfunction. Our nightmare induced case of TTS is unique in presentation and is also notable as a recurrence of TTS while on a beta-1 selective agent. We also believe that there may be physiologic and clinical benefit of utilizing nonspecific beta-blockers in treatment of TTS. However use of beta blocking agents in TTS has not been validated and additional research is needed to guide management and reduce recurrence of TTS.

\section{References}

1. Ghadri JR, Wittstein IS, Prasad A, Sharkey S, Dote K, et al. (2018) International expert consensus document on takotsubo syndrome (Part I): Clinical Characteristics, Diagnostic Criteria, and Pathophysiology. Eur Heart J 39: 2032-2046.

2. Scantlebury DC, Prasad A, Prasad A (2014) Diagnosis of takotsubo cardiomyopathy - Mayo Clinic criteria. Circulation Journal 78: 2129-2139.

3. Murdock RW, Murdock DK (2016) Clinical characterization of pharmacologically induced takotsubo syndrome: Implications for treatment and mechanisms. Int $\mathrm{J}$ Clin Cardiol 3: 078.

4. Lyon AR, Bossone E, Schneider B, Sechtem U, Citro R, et al. (2015) Current state of knowledge on Takotsubo syndrome: A Position Statement from the Taskforce on Takotsubo Syndrome of the Heart Failure Association of the European Society of Cardiology. Eur J Heart Fail 18: 8-27.

5. Haghi D, Papavassiliu T, Heggemann F, Kaden JJ, Borggrefe M, et al. (2008) Incidence and clinical significance of left ventricular thrombus in tako-tsubo cardiomyopathy assessed with echocardiography. QJM 101: 381-386.

6. Sharkey SW, Windenburg DC, Lesser JR, Maron MS, Hauser RG, et al. (2010) Natural history and expansive clinical profile of stress (tako-tsubo) cardiomyopathy. J Am Coll Cardiol 55: 333-341.

7. Shin SN, Yun KH, Ko JS, Rhee SJ, Yoo NJ, et al. (2011) Left ventricular thrombus associated with takotsubo cardiomyopathy: A cardioembolic cause of cerebral infarction. J Cardiovasc Ultrasound 19: 152-155.

8. Cecchi E, Parodi G, Giglioli C, Passantino S, Bandinelli B, et al. (2013) Stress-induced hyperviscosity in the pathophysiology of takotsubo cardiomyopathy. Am J Cardiol 111: 1523-1529. 
9. Tobar R, Rotzak R, Rozenman Y (2009) Apical thrombus associated with Takotsubo cardiomyopathy in a young woman. Echocardiography 26: 575-580.

10. Shridhar P, Omran S, Hajjali R, Lasorda D, Khalil R, et al. (2016) Transient mid-ventricular ballooning due to bad dream in a postmenopausal woman. World Journal of Cardiovascular Diseases 6: 329-332.

11. Song BG, Oh JH, Kim HJ, Kim SH, Chung SM, et al. (2013) Chronobiological variation in the occurrence of Tako-tsubo cardiomyopathy: Experiences of two tertiary cardiovascular centers. Heart Lung 42: 40-47.
12. Citro R, Previtali M, Bovelli D, Vriz O, Astarita C, et al. (2009) Chronobiological patterns of onset of Tako-Tsubo cardiomyopathy: A multicenter Italian study. J Am Coll Cardiol 54: 180-181.

13. Kato K, Di Vece D, Cammann VL, Micek J, Szawan KA, et al. (2019) Takotsubo recurrence: Morphological types and triggers and identification of risk factors. J Am Coll Cardiol 73: $982-984$.

14. Kato K, Lyon AR, Ghadri JR, Templin C (2017) Takotsubo syndrome: Aetiology, presentation and treatment. Heart 103: 1461-1469. 